\title{
Effect of pre-anesthetic anxiety and heart rate on propofol dose requirement for induction: A correlation study
}

\author{
Manjunatha S M1', Rajdip Hazra², Ashok Das ${ }^{3}$, Sisir Chakraborty ${ }^{4}$, Rajarshi Bose ${ }^{5}$, \\ Swadesh Garain ${ }^{6}$, Debaditya Purkait ${ }^{7}$ \\ ${ }^{1}$ Assistant Professor, Department of Anesthesiology, Bangalore Medical College and Research Institute, Bangalore, \\ Karnataka, ${ }^{2}$ Tutor, ${ }^{7}$ Post Graduate Trainee, Department of Anesthesiology, Nilratan Sircar Medical College and \\ Hospital, ${ }^{3}$ Assistant Professor, Department of Anesthesiology, ESIC Medical College and Hospital, Joka, ${ }^{4}$ Assistant \\ Professor, Department of Medicine, College of Medicine and Sagore Dutta Hospital, ${ }^{5}$ Assistant Professor, Department \\ of Pediatric Medicine, Nilratan Sircar Medical College and Hospital, Kolkata, ${ }^{6}$ Specialist Medical Officer, Department of \\ Gynecology and Obstetrics, Nadia District Hospital, Krisnanagar, West Bengal, India
}

A B S T R A C T

Background: Though anxiety is associated with increase in heart rate and cardiac output, there is conflicting reports regarding its effect on propofol induction dose. Aims and Objectives: The aim of the study was to delineate the correlation between pre-anesthetic anxiety and heart rate on propofol requirement for induction. Materials and Methods: Total 42 patients of ASA (American Society of Anesthesiologists) physical status I and II, 13 male and 29 female, aged between 18 to 50 years and scheduled for elective surgery under general anesthesia, were enrolled in this study. Trait anxiety in the waiting room and state anxiety both in the waiting room and operating room were assessed using Spielberger's revised State-Trait Anxiety Inventory (STAI) scale. After heart rate (HR) recording, anesthesia was induced with $200 \mathrm{ml} / \mathrm{hr} 1 \%$ propofol infusion till loss of verbal contact. Results: Pre-anesthetic trait anxiety and HR had strong positive correlation with propofol requirement $(\rho>0.6, P<0.05)$ while state anxiety had not. But pre-anesthetic state anxiety measured just before induction is strongly associated with increase in $\mathrm{HR}(\rho>0.6, P<0.05)$. Conclusion: Pre-operative anxiety should be considered and the dose of propofol should be titrated for induction accordingly. Further, larger trials with more sophisticated monitoring are needed to confirm our findings.

Key words: Anxiety, Heart rate, p ropofol, Induction

\section{INTRODUCTION}

Anxiety is a very common phenomenon during peri-operative period. Pre-operative anxiety is described as an unpleasant state of uneasiness or tension that is secondary to a patient being concerned about a disease, hospitalization, anesthesia and surgery, or the unknown. ${ }^{1}$ It creates a great discomfort to the patient. Previous studies have shown that patients with higher anxiety not only experience more intraoperative hemodynamic disturbances, post operative pain and recovery time but also require more anesthetic drugs. ${ }^{2,3}$ The studies have been conducted on different hypnotic drugs from methohexitone to propofol for the purpose to test the hypothesis of whether preoperative anxiety actually increases the requirement of these hypnotic drugs. ${ }^{4-7}$ At present Propofol is the most frequently investigated hypnotic drug and have been proved to follow the same hypothesis of higher the anxiety, higher the amount of drug required. ${ }^{5,8}$ But some recent studies have come with different results. ${ }^{9,10}$

Anxiety and acute mental stress are associated with increased heart rate (HR) and cardiac output ${ }^{11,12}$ and studies have shown that increased cardiac output increases the propofol requirements. ${ }^{13-16}$ Hence hemodynamic status during induction of anesthesia may modify propofol 
requirements. ${ }^{13-18}$ In other words, stabilization of hemodynamic status may limit effect of pre-anesthetic anxiety on propofol dose requirement for induction.

We designed this study to address the effect of pre-anesthetic anxiety and heart rate on propofol dose requirement for induction; and the effect of pre-anesthetic anxiety on heart rate. Loss of verbal contact was defined as the end point for induction.

\section{MATERIALS AND METHODS}

After approval from institutional ethical committee and obtaining written informed consent of the patients, the study was conducted in Nilratan Sircar Medical College and Hospital, a tertiary care medical college hospital in eastern India. Total 42 patients of ASA (American Society of Anesthesiologists) physical status I and II, 13 male and 29 female, aged between 18 to 50 years and scheduled for elective surgery under general anesthesia, were enrolled in this study. Power calculations suggested that a minimum of 21 subjects would be required for the study to reject or accept the null hypothesis [assuming study power of $90 \%$, probability of type I error (alpha) of $5 \%$ and minimum positive Pearson's correlation coefficient (rho) of 0.6]. To be on a safer side, a total of 42 patients were incorporated for the study $(n=42)$.

Pregnant patients and those with anemia (defined as hemoglobin less than $10 \mathrm{gm} \%$ ); cardiovascular disease; neurologic or psychiatric disease; chronic medication with beta-blockers, anxiolytics, antidepressants, opioids; documented alcohol or drug abuse and thyroid patients (both hypo and hyperthyroid) were excluded from the study. Patients unable to communicate or complete the anxiety forms (e.g., deaf and dumb patients) were also excluded from the study.

On the day before surgery during pre-anesthetic evaluation, proper history was taken and examination was done. Anxiety assessment was done using Spielberger's revised State-Trait Anxiety Inventory (STAI) scale or form Y. The STAI is a widely used self-report anxiety assessment instrument with two subscales each containing 20 statements. Statements 1-20 measure situational or state anxiety (STAI-S), and 21-40 measure underlying or trait anxiety (STAI-T). For STAI-S, respondents are asked to indicate "how you feel right now, that is, at this moment". Responses indicate intensity of feeling on a 1 to 4 scale, from "not at all" through "somewhat", "moderately so" to "very much so". For STAI-T the question concerns "how you generally feel" and the response scale indicates frequency: "almost never", "sometimes", "often" and "almost always". After reversing scores for positively-worded items, total scores for STAI-S and STAI-T are calculated, ranging from $20-80$. Both scales were intended to form uni-dimensional measures.

In the morning of surgery, while waiting in their room in the surgical ward, patients were asked to complete the 2 STAI forms, i.e., STAI-S and STAI-T. On arrival in the operating room, an 18-gauge intravenous (IV) catheter was inserted. A 3-way stopcock was connected to the IV catheter and the Propofol infusion was connected. ASA standard monitors were attached. Patients were monitored with non-invasive arterial blood pressure (NIBP), 5-lead electrocardiogram (ECG), pulse oximetry and temperature probe. No preanesthetic medication was given to the patient. Just before the induction of anesthesia, patients were asked again to complete STAI-S form. Once the patients had completed STAI-S form, HR were measured (mean of 3 consecutive measurements taken 1 minute apart).

General anesthesia was induced with a continuous Propofol infusion (1\% Propofol at $200 \mathrm{ml} / \mathrm{hr})$. End point of induction was defined as loss of verbal response. From the start of Propofol infusion, patients were asked to say "ONE", in a normal voice but without tactile stimulation, each time when we ask to say the word. In this way, verbal response was tested every 15 seconds. Immediately the patient first failed to respond, the volume displayed on the pump was recorded as Propofol dose required for induction and the study was terminated. Propofol infusion was continued in a maintenance dose $(6 \mathrm{mg} / \mathrm{kg} / \mathrm{hr})$, premedications (opioid, anti-sialogogue etc.) given and general anesthesia continued in a standard fashion.

During the study, data were collected by an investigator who was blinded for STAI results.

\section{Statistical analysis}

Continuous numerical data were expressed as median, inter-quartile range and range (extreme values). Analysis was conducted with SPSS software version 18.0 (SPSS Inc., Chicago, Illinois, USA). Associations between STAI-T and STAI-S in the waiting room, STAI-S and HR in the operating room versus Propofol dose for induction were studied with the Spearman test. Association between STAI-S in the operating room versus HR was also studied with the Spearman test. All tests were two tailed. A $p$ value of less than 0.05 was considered as statistically 'significant' $(p<0.05)$.

\section{RESULTS}

Total 42 patients, 13 male and 29 female, aged between 18 to 50 years, were enrolled in this study. There characteristics are presented in 'Table 1' as median, interquartile range and range. No patient was excluded from the study. 
STAI forms were measured in the waiting room in the ward (both STAI-T and STAI-S), again just before the operation (STAI-S only) with a time gap of 2 hours. All the patients were given exactly 2 hrs gap between these two measurements. Upon induction, the median propofol dose was $110 \mathrm{mg}$ (inter-quartile range 100-124 mg; range: 72-142 mg).

'Table 2' shows the association between the STAI-T in the waiting room, STAI-S in the waiting room, STAI-S in the operating room and $\mathrm{HR}$ in the operating room with the propofol dose required for induction.

While STAI-T in the waiting room showed a strong positive correlation with Propofol dose required for induction (Figure 1), STAI-S had no significant positive correlation, both in the waiting room and in the operating room (Figures 2 and 3). On the other hand, we observed a strong positive correlation between HR in the operating room and induction dose of Propofol (Figure 4). We also observed a strong positive correlation between STAI-S in operating room and HR (Table 3 and Figure 5) [cut off for minimum positive Pearson's correlation coefficient was set at 0.6 , as described in the methodology].

\section{DISCUSSION}

Our study was carried out to observe the correlation between pre-anesthetic anxiety and heart rate on Propofol dose requirement. Pre-anesthetic anxiety was studied under two components of anxiety i.e., state and trait using Spielberger's State-Trait Anxiety Inventory Scale (STAI-S and STAI-T respectively). State anxiety refers to

\begin{tabular}{lccc}
\multicolumn{4}{l}{ Table 1: Patient characteristics } \\
\hline $\begin{array}{l}\text { Patient } \\
\text { characteristics }\end{array}$ & Median & $\begin{array}{c}\text { Inter-quartile } \\
\text { range (1:3) }\end{array}$ & Range \\
\hline Age (years) & 37 & $28-45$ & $18-50$ \\
Weight (Kg) & 56 & $51-60$ & $36-70$ \\
STAI-T in waiting room & 37 & $34-38$ & $30-49$ \\
STAI-S in waiting room & 40 & $37-44$ & $33-53$ \\
STAI-S in operating & 42 & $39-46$ & $33-54$ \\
room & & & \\
HR in operating room & 115 & $108-123$ & $68-132$ \\
\hline
\end{tabular}

\begin{tabular}{lcc}
$\begin{array}{l}\text { Table 2: Correlation coefficient of different } \\
\text { study parameters against the induction dose of } \\
\text { propofol }\end{array}$ & \multicolumn{2}{c}{ Propofol dose } \\
\hline Patient characteristics & $\boldsymbol{\rho}$ (rho) & $\boldsymbol{p}$ value \\
\hline & 0.673 & $<0.0001$ \\
\hline STAI-T in waiting room & 0.478 & 0.0012 \\
STAI-S in waiting room & 0.522 & 0.0001 \\
STAI-S in operating room & 0.662 & $<0.0001$ \\
HR in operating room & \multicolumn{2}{l}{} \\
\hline$\rho($ rho) = Pearson's correlation coefficient & &
\end{tabular}

transitory unpleasant feelings of apprehension, tension, nervousness or worry, often accompanied by activation of the autonomic nervous system; it reflects how threatening a person perceives his environment to be. ${ }^{19}$ Trait anxiety is a personality disposition that describes a person's tendency to perceive situations as threatening, and hence to experience state anxiety in stressful situations. ${ }^{19}$ Trait anxiety is not observed directly, but is expressed as state anxiety when stress is experienced. Hence we studied the effect of trait and state anxiety on propofol requirement separately.

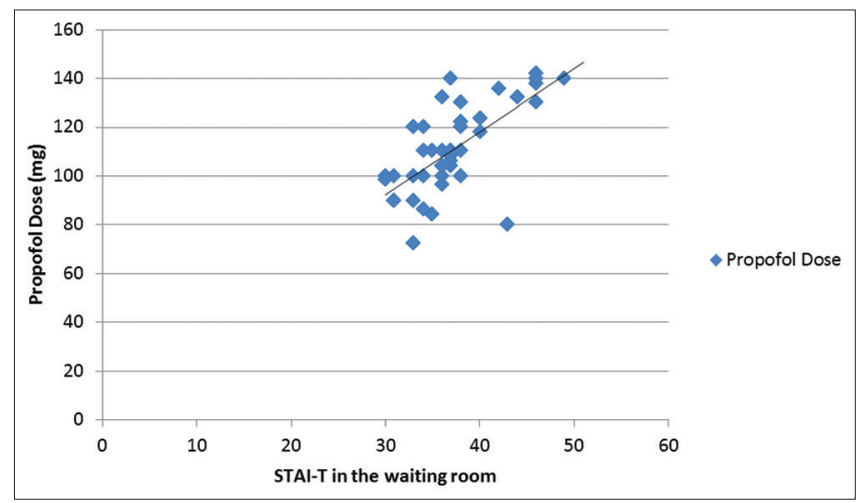

Figure 1: Scatter diagram showing relationship between STAI-T in waiting room and propofol dose

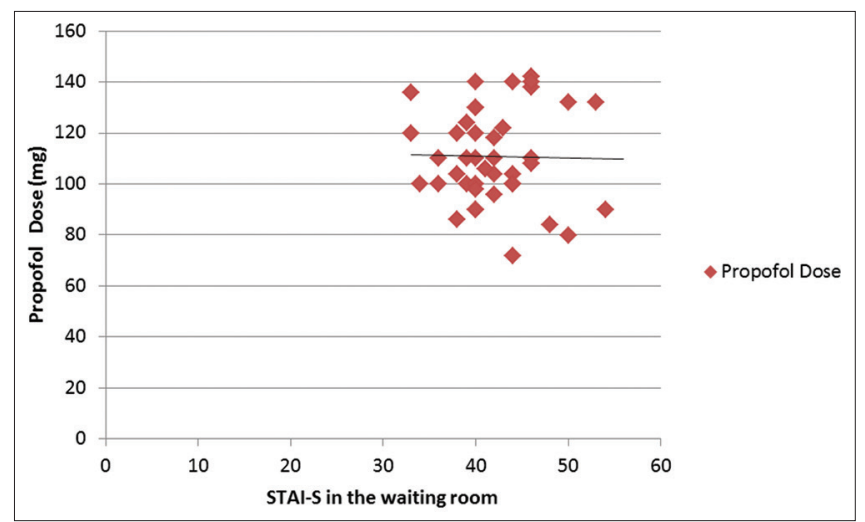

Figure 2: Scatter diagram showing relationship between STAI-S in waiting room and propofol dose

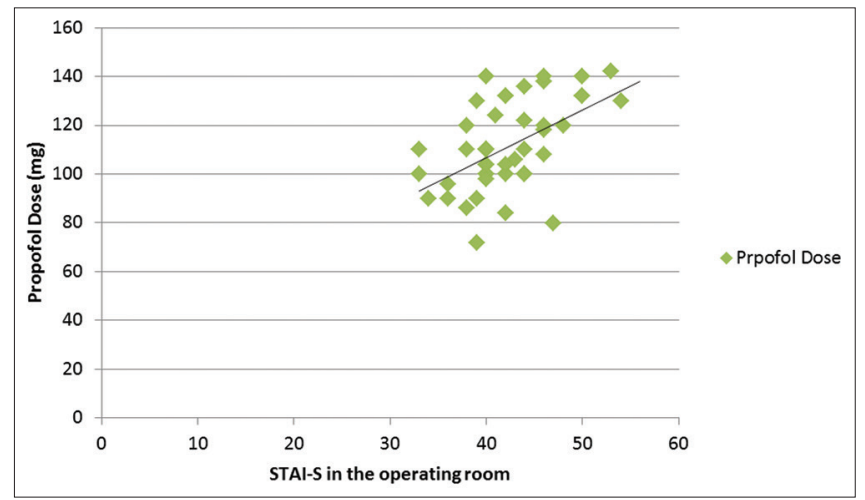

Figure 3: Scatter diagram showing relationship between STAI-S in operating room and propofol dose

Asian Journal of Medical Sciences | Mar-Apr 2017 | Vol 8 | Issue 2 
Table 3: Correlation coefficient of STAI-S in the operating room against HR

\begin{tabular}{lll}
\hline Patient characteristics & \multicolumn{2}{c}{ Heart Rate (HR) } \\
\cline { 2 - 3 } & $\boldsymbol{\rho}$ (rho) & $\boldsymbol{p}$ value \\
\hline STAI-S in operating room & 0.606 & $<0.0001$ \\
\hline$\rho($ rho $)=$ Pearson's correlation coefficient & &
\end{tabular}

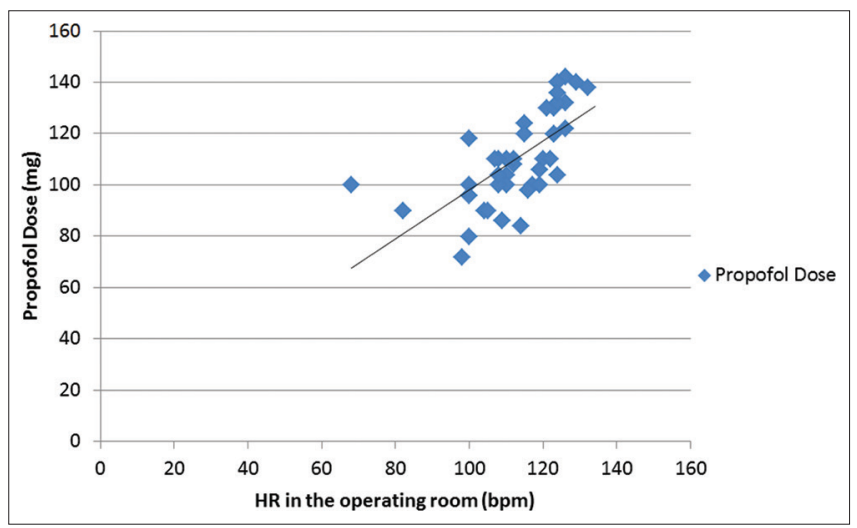

Figure 4: Scatter diagram showing relationship between HR in operating room and propofol dose

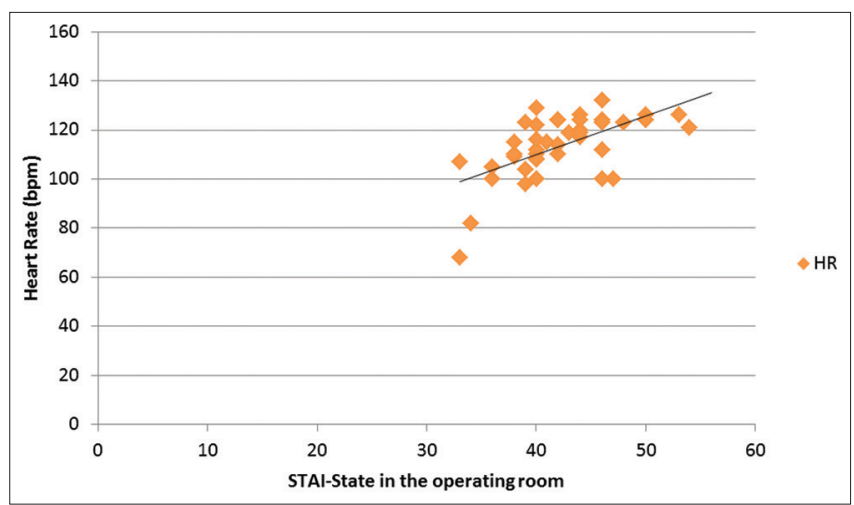

Figure 5: Scatter diagram showing relationship between STAI-S and $H R$ in operating room

We also studied the effect of anxiety on HR and HR on propofol dose requirement.

We observed that preoperative trait anxiety (STAI-T), which was measured in the waiting room, independently affected the Propofol dose required for induction (Figure 1). But the state anxiety (STAI-S), measured both in the waiting room (Figure 2) and in the operating room just before induction (Figure 3), had no significant positive correlation with the Propofol dose requirement. This is in agreement with previous studies of Marantes et al. ${ }^{5}$ and Kil et al. ${ }^{6}$

On the other hand, heart rate (HR), which was measured in the operating room just before the induction (Figure 4), had strong positive correlation with the Propofol dose requirement for induction. We also observed that STAI-S measured in the operating room had a strong positive correlation with HR (Figure 5). This is in agreement with Morely et al. ${ }^{9}$ and Gras S et al. ${ }^{10}$

Conflicting results have been reported in the previous studies concerning the effects of anxiety on Propofol dose requirement for induction. Maranets et al. found that increased preoperative STAI-T on the day of surgery was associated with higher Propofol bolus dose to achieve a BIS (bispectral index) value between 40 and $60 .{ }^{5}$ Kil et al. reported that, during the induction of anesthesia, patients with higher levels of both state and trait anxieties required greater amounts of propofol to reach light (85) and moderate levels (75) of sedation. ${ }^{6}$ However, the amount of Propofol required to reach a deep level of sedation (BIS 65), was associated with higher levels of trait, but not state anxiety.

On the contrary, Morley et al. did not observe any influence of the STAI-T or STAI-S with propofol dose administered to achieve a BIS of $50 .{ }^{9}$ Gras $\mathrm{S}$ et al. found no strong correlation between state and trait anxiety with propofol dose requirement for loss of consciousness (LOC). ${ }^{10}$ They found significant positive correlation between HR in the operating room and propofol dose and also between STAI-S in the operating room and HR, thereby supporting an indirect effect of pre-anesthetic anxiety through changes in the HR.

In our study, we found strong positive correlation between trait anxiety and Propofol dose requirement and also between HR and the Propofol dose requirement with a $\mathrm{p}$-value of $<0.05$. This is in contrary with Gras $\mathrm{S}$ et al. who found strong positive correlation only between HR and Propofol dose requirement. ${ }^{10}$ It has been proven a long back that anxiety is associated with a raise in both HR and cardiac output $(\mathrm{CO}){ }^{11,12,20}$ Fell et al. suggested that anxiety and stress-induced adrenaline release may account for the preoperative increase in $\mathrm{HR}$ and $\mathrm{CO} .{ }^{21}$ Anxiety causes increase in catecholamine levels through sympathetic surge. Catecholamines affect CO by increasing contractility and HR. Hence Propofol dose requirement is increased in anxiety as there is increase in CO by increase in both HR and contractility.

Here we have chosen loss of verbal contact as our clinical end point, because it is the commonly accepted terminal point used in many studies of the effects of hypnotic drugs. ${ }^{12,16}$ We chose to administer a slow continuous infusion to minimize measurement error because LOC was assessed clinically at intervals of 15 seconds.

However, there were several limitations of this study. Anxiety measurement was based on subjective scorings of anxiety rather than objective physical indicators. Hence, self reporting bias might present. But the STAI is the best scoring method available till now used in most of the related studies. ${ }^{5-7,10}$ 
It would have been more accurate if we had used propofol arterial concentrations and cardiac output measurements. Use of BIS or any other EEG (electroencephalogram) analog/depth indicators would have been helpful in confirming the level of depth of anesthesia. This was merely a correlation study and from the present data we can not conclude that increase in propofol requirement is related to pharmacodynamic or pharmacokinetic changes. Another aspect was the inclusion of nearly $70 \%$ female subjects as study population. There are studies both supporting and opposing female preponderance of pre-operative anxiety. ${ }^{22}$ Among other limitations were small sample size, single-center design and selected study population (pediatric, elderly and ASA III-IV patients were excluded).

\section{CONCLUSION}

Our present study showed that pre-anesthetic trait anxiety and heart rate have direct effect on dose of propofol requirement for induction, while the state anxiety in the operating room by acting through heart rate might indirectly influence propofol requirement for induction. Hence pre-operative anxiety should be considered and the dose of propofol should be titrated for induction accordingly. Further, larger trials with more sophisticated monitoring are needed to confirm our findings.

\section{ACKNOWLEDGEMENTS}

The authors like to acknowledge Nilratan Sircar Medical College and Hospital, Kolkata, India for providing necessary support during this research work. None of the authors have any conflict of interest.

\section{REFERENCES}

1. Ramsay MAE. A survey of pre-operative fear. Anaesthesia 1972; 27(4):396-402.

2. Johnston M. Pre-operative emotional states and post-operative recovery. Adv Psychosom Med 1986; 15:1-22.

3. Wallace LM. Trait anxiety as a predictor of adjustment to and recovery from surgery. Br J Clin Psychol 1987; 26(1):73-74.

4. Goldmann L, Ogg TW and Levey AB. Hypnosis and daycase anaesthesia: A study to reduce pre-operative anxiety and intra-operative anaesthetic requirements. Anaesthesia 1988; 43(6):466-469.

5. Marantes I and Kain ZN. Preoperative anxiety and intraoperative anesthetic requirements. Anesth Analg 1999; 89(6):1346-1351.
6. Kil HK, Kim WO, Chung WY, Kim GH, Seo H and Hong JY. Preoperative anxiety and pain sensitivity are independent predictors of propofol and sevoflurane requirements in general anaesthesia. Br J Anaesth 2012; 108(1):119-125.

7. Osborn TM and Sandler NA. The effects of preoperative anxiety on intravenous sedation. Anesth Prog 2004; 51(2):46-51.

8. Hong JY, Kang IS, Koong MK, Yoon HJ, Jee YS, Park JW, et al. Preoperative anxiety and propofol requirement in conscious sedation for ovum retrieval. J Korean Med Sci 2003; 18(6):863-868.

9. Morley AP, Papageorgiou $\mathrm{CH}$, Marinaki AM, Cooper DJ and Lewis CM. The effect of pre-operative anxiety on induction of anaesthesia with propofol. Anaesthesia 2008; 63(5):467-473.

10. Gras S, Servin F, Bedairia E, Montravers P, Desmonts JM, Longrois $\mathrm{D}$, et al. The effect of preoperative Heart rate and anxiety on the propofol dose required for loss of consciousness. Anesth Analg 2010; 110(1):89-93.

11. Piccirillo G, Elvira S, Bucca C, Viola E, Cacciafesta M and Marigliano V. Abnormal passive head-up tilt test in subjects with symptoms of anxiety power spectral analysis study of heart rate and blood pressure. Int J Cardiol 1997; 60(2):121-131.

12. Tenenbaum $G$ and Milgram RM. Trait and state anxiety in Israeli student athletes. J Clin Psychol 1978; 34:691-693.

13. Upton RN, Ludbrook GL, Grant $C$ and Martinez A. Cardiac output is a determinant of the initial concentrations of propofol after short-infusion administration. Anesth Analg 1999; 89(3):545-552.

14. Johnson KB, Egan TD, Kern SE, White JL, McJames SW, Syroid $N$, et al. The influence of hemorrhagic shock on propofol: A pharmacokinetic and pharmacodynamic analysis. Anesthesiology 2003; 99(2):409-420.

15. Myburgh JA, Upton RN, Grant C and Martinez A. Epinephrine, norepinephrine and dopamine infusions decrease propofol concentrations during continuous propofol infusion in an ovine model. Intensive Care Med 2001; 27(1):276-282.

16. Adachi $\mathrm{YU}$, Watanabe $\mathrm{K}$, Higuchi $\mathrm{H}$ and Satoh $\mathrm{T}$. The determinants of propofol induction of anesthesia dose. Anesth Analg 2001; 92(3):656-661.

17. Wilson ES, McKinlay S, Crawford JM and Robb HM. The influence of esmolol on the dose of propofol required for induction of anaesthesia. Anaesthesia 2004; 59(2):122-126.

18. Takizawa E, Takizawa D, Al-Jahdari WS, Miyazaki M, Nakamura K, Yamamoto K, et al. Influence of Atropine on the dose requirements of propofol in humans. Drug Metab Pharmacokinet 2006; 21(5):384-388.

19. Gaudry E, Vagg P and Spielberger CD. Validation of the statetrait distinction in anxiety research. Multivariate Behav Res 1975; 10(3):331-341.

20. Bergamaschi $M$ and Longoni AM. Cardiovascular events in anxiety: Experimental studies in the conscious dog. Am Heart J 1973; 86:385-394.

21. Fell D, Derbyshire DR, Maile CJD, Larsson IM, Ellis R, Achola KJ, et al. Measurement of plasma catecholamine concentrations: An assessment of anxiety. Br J Anaesth 1985; 57(8):770-774.

22. Seifu Nigussie, Tefera Belachew and Wadu Wolancho. Predictors of preoperative anxiety among surgical patients in Jimma University Specialized Teaching Hospital, South Western Ethiopia. BMC Surg 2014; 14:67. 